Draft version August 22, 2018

Preprint typeset using $\mathrm{IAT}_{\mathrm{E}} \mathrm{X}$ style emulateapj v. 5/2/11

\title{
A MASSIVE PROTOSTAR FORMING BY ORDERED COLLAPSE OF A DENSE, MASSIVE CORE
}

\author{
Yichen Zhang ${ }^{1}$, Jonathan C. Tan ${ }^{1,2}$, James M. De Buizer ${ }^{3}$, Göran Sandell ${ }^{3}$, Maria T. Beltran ${ }^{4}$, Ed \\ Churchwell $^{5}$, Christopher F. McKee ${ }^{6,7}$, Ralph Shuping ${ }^{3}$, Jan E. Staff ${ }^{8}$, Charles Telesco ${ }^{1}$, Barbara Whitney ${ }^{5}$ \\ ${ }^{1}$ Department of Astronomy, University of Florida, Gainesville, Florida 32611, USA \\ ${ }^{2}$ Department of Physics, University of Florida, Gainesville, Florida 32611, USA \\ ${ }^{3}$ SOFIA-USRA, NASA Ames Research Center, MS 232-12, Building N232, P. O. Box 1, Moffett Field, CA 94035, USA \\ ${ }^{4}$ INAF-Osservatorio Astrofisico di Arcetri, Largo E. Fermi 5, Firenze I-50125, Italy \\ ${ }^{5}$ Department of Astronomy, University of Wisconsin, Madison, Wisconsin 53706, USA \\ ${ }^{6}$ Department of Astronomy and Astrophysics, University of California, Berkeley, California 94720, USA \\ ${ }^{7}$ Department of Physics, University of California, Berkeley, California 94720, USA \\ ${ }^{8}$ Department of Physics and Astronomy, Louisiana State University, Baton Rouge, Louisiana 70803, USA \\ yc.zhang@astro.ufl.edu \\ Draft version August 22, 2018
}

\begin{abstract}
We present 30 and $40 \mu \mathrm{m}$ imaging of the massive protostar G35.20-0.74 with SOFIA-FORCAST. The high surface density of the natal core around the protostar leads to high extinction, even at these relatively long wavelengths, causing the observed flux to be dominated by that emerging from the near-facing outflow cavity. However, emission from the far-facing cavity is still clearly detected. We combine these results with fluxes from the near-infrared to $\mathrm{mm}$ to construct a spectral energy distribution (SED). For isotropic emission the bolometric luminosity would be $3.3 \times 10^{4} L_{\odot}$. We perform radiative transfer modeling of a protostar forming by ordered, symmetric collapse from a massive core bounded by a clump with high mass surface density, $\Sigma_{\mathrm{cl}}$. To fit the SED requires protostellar masses $\sim 20-34 M_{\odot}$ depending on the outflow cavity opening angle $\left(35^{\circ}-50^{\circ}\right)$, and $\Sigma_{\mathrm{cl}} \sim 0.4-1 \mathrm{~g} \mathrm{~cm}^{-2}$. After accounting for the foreground extinction and the flashlight effect, the true bolometric luminosity is $\sim(0.7-2.2) \times 10^{5} L_{\odot}$. One of these models also has excellent agreement with the observed intensity profiles along the outflow axis at 10, 18, 31 and $37 \mu \mathrm{m}$. Overall our results support a model of massive star formation involving the relatively ordered, symmetric collapse of a massive, dense core and the launching bipolar outflows that clear low density cavities. Thus a unified model may apply for the formation of both low and high mass stars.
\end{abstract}

Subject headings: stars: formation

\section{INTRODUCTION}

Massive stars impact many areas of astrophysics. In most galactic environments they dominate the radiative, mechanical and chemical feedback on the interstellar medium, thus regulating the evolution of galaxies. Many low-mass stars form in clusters along with massive stars, and their protoplanetary disks can be affected by this feedback also. There is some evidence that our own solar system was influenced in this way (Adams 2010). Despite this importance, there is no consensus on the basic formation mechanism of massive stars. Theories range from Core Accretion, i.e., a scaled-up version of lowmass star formation (e.g. the Turbulent Core Model of McKee \& Tan 2003, MT03), to Competitive Accretion at the centers of forming star clusters (Bonnell et al. 2001, Wang et al. 2010), to Stellar Collisions (Bonnell et al. 1998).

The Core Accretion theory predicts the existence of an envelope-fed, central accretion disk and relatively ordered and collimated bipolar outflows powered by accretion around a massive protostar. The other formation mechanisms predict a much less ordered geometry of gas and dust surrounding the protostar.

Collimated bipolar outflows have been observed from massive protostars (e.g. Beuther et al. 2002). Outflows may limit the formation efficiency (mass ratio of final star to initial gravitationally-bounded protostellar core) to $\sim 0.5$ (Matzner \& McKee 1999), since they expel ma- terial along polar directions. The resulting low-density cavities have been proposed to significantly affect the appearance of the protostar in the MIR (De Buizer 2006, De Buizer et al. 2012), and this is seen in the radiative transfer (RT) calculations of Zhang \& Tan (2011, ZT11) and Zhang et al. (2013, ZTM13) using the RT code developed by Whitney et al. (2003).

The Turbulent Core Model also relates core properties to the surrounding self-gravitating star-cluster-forming clump. For a marginally unstable pre-stellar core of a given mass, its size, density and subsequent accretion rate all depend on the pressure set by the mean mass surface density of the clump, $\Sigma_{\mathrm{cl}}$. Observed values of $\Sigma_{\mathrm{cl}}$ are $\sim 0.1-1 \mathrm{~g} \mathrm{~cm}^{-2}$ (MT03; Butler \& Tan 2012), leading to high predicted accretion rates, $\sim 10^{-4}-10^{-3} M_{\odot} \mathrm{yr}^{-1}$, potentially important for overcoming the high radiation pressure from the massive protostar.

G35.20-0.74 (hereafter G35.2) is a massive protostar associated with a well-defined outflow. At a distance of $2.2 \mathrm{kpc}$ (Zhang et al. 2009), radio continuum emission shows that it contains an ultracompact HII region at the center in the form of a collimated jet along the north-south direction (Heaton \& Little 1988, Gibb et al. 2003), which may be an example of an Outflow-Confined HII Region (Tan \& McKee 2003). The outflow is also observed in the NIR (Fuller et al. 2001) with the northern cavity brighter than the southern one. Ground-based 10 and $18 \mu \mathrm{m}$ observations only reveal the northern cavity 
(De Buizer 2006), suggesting it is inclined towards us. Outflow is also seen in CO Gibb et al. 2003, Birks et al. 2006), including a wider angle flow in the NE-SW direction. It is unclear if this results from a separate driving source or is related to the N-S jet, but misaligned by interaction with the surrounding core/clump. In previous studies, an early B star was thought to be present at the center (Dent et al. 1985), surrounded by an envelope with mass $\sim 500 M_{\odot}($ Little et al. 1998) $)$ to $\sim 3800 M_{\odot}$ (Paron \& Weidmann 2010).

Here we present the first SOFIA-FORCAST images of G35.2. Combined with other multiwavelength data, we construct the SED. We present a simple exploration of parameter space of RT models of massive protostars and use the observed SED and intensity profiles along the outflow axis to constrain the models.

\section{OBSERVATIONS \& DATA REDUCTION}

The SOFIA-FORCAST (Herter et al. 2012) observations of G35.2 were performed on May 24 and 26 (UT) 2011 in the $11.3,19.7,31.5$, and $37.1 \mu \mathrm{m}$ filters at an altitude of $43000 \mathrm{ft}$. The chopping secondary of SOFIA was driven at $4 \mathrm{~Hz}$, with a matched chop and nod throw of $60^{\prime \prime}$, and with a nod performed every 30s. The final effective on-source exposure times were $909 \mathrm{~s}$ at $11.3 \mu \mathrm{m}$, $959 \mathrm{~s}$ at $19.7 \mu \mathrm{m}, 4068 \mathrm{~s}$ at $31.5 \mu \mathrm{m}$, and $4801 \mathrm{~s}$ at $37.1 \mu \mathrm{m}$. The fluxes were calibrated by the SOFIA data reduction pipeline, which during the Basic Science period has an estimated $3 \sigma \leq 20 \%$ calibration error in all filters (Herter et al. 2012).

The 11 and $31 \mu \mathrm{m}$ images were observed simultaneously with the dichroic mode of FORCAST; and similarly for the 19 and $37 \mu \mathrm{m}$ images. The relative offsets between the short and long wavelength arrays are known to better than $1 \sigma=0.15^{\prime \prime}$. The best registration of the $19 \mu \mathrm{m}$ image with respect to the $18.3 \mu \mathrm{m}$ image from De Buizer (2006) was obtained using a $\chi^{2}$ minimization algorithm. The latter was convolved with a Gaussian profile to reach the same resolution of the FORCAST image. Several features in the two images match up well after the registration increasing confidence in the technique. We adopt the pixel size $\left(0.768^{\prime \prime}\right)$ as our conservative $1 \sigma$ uncertainty in the relative offset between the 18.3 and $19.7 \mu \mathrm{m}$ images. Considering the absolute astrometric uncertainties of $\sim 0.2^{\prime \prime}$ from the $18.3 \mu \mathrm{m}$ image, we get an absolute astrometric uncertainty of $0.79^{\prime \prime}$ from the $19.7 \mu \mathrm{m}$ image. The absolute astrometric uncertainty of the $37.1 \mu \mathrm{m}$ image is then estimated to be better than $0.8^{\prime \prime}$. However, the $11.3 \mu \mathrm{m}$ FORCAST image suffered from such low $\mathrm{S} / \mathrm{N}$ that it could not be used to perform similar registration for the $31.4 \mu \mathrm{m}$ image. We had to use the $\chi^{2}$ minimization algorithm to find the best registration of the $31.4 \mu \mathrm{m}$ image and the astrometrically calibrated $37.1 \mu \mathrm{m}$ image, noticing that they look remarkably similar morphologically. The absolute astrometric uncertainty in the $31.4 \mu \mathrm{m}$ image is estimated to be $0.89^{\prime \prime}$.

Using standard stars observed throughout several Basic Science flights, an average FWHM for each wavelength was determined: $3.9^{\prime \prime}$ at $19.7 \mu \mathrm{m}, 4.1^{\prime \prime}$ at $31.5 \mu \mathrm{m}$, and $4.5^{\prime \prime}$ at $37.1 \mu \mathrm{m}$. The images were then deconvolved using the maximum likelihood method (Richardson 1972, Lucy 1974), which yielded an approximately factor of two better resolution than the pre-deconvolved images. The deconvolved images compare favorably to simple unsharp masking of the original images, and in the case of the $19.7 \mu \mathrm{m}$ image, it compares well to the Gemini $18.3 \mu \mathrm{m}$ image, and thus the substructures revealed in the deconvolved images are believed to be real.

We also retrieved calibrated level 2 data (AOR 1342241157, HOBYS GT program) from HerschelSPIRE and a single long integration SCUBA 850/450 $\mu \mathrm{m}$ jiggle-map form the JCMT archive at CADC1. The SCUBA data were reduced and calibrated in a standard way using SURF (Sandell et al. 2001).

\section{RESULTS}

Fig. (11) shows the FORCAST images at 31.5 and $37.1 \mu \mathrm{m}$ along with the 11 and $18 \mu \mathrm{m}$ images by GeminiT-ReCS (De Buizer 2006) and 3.6 and $8 \mu \mathrm{m}$ images by Spitzer-IRAC (GLIMPSE Survey, Churchwell et al. 2009). The airborne FORCAST images have a dynamic range of $\sim 4$ orders of magnitude, similar to the space-based IRAC observations and much higher than the ground-based T-ReCS observations. The origin of the images is chosen to be the radio source G35.2N (R.A. $=18 \mathrm{~h} 58 \mathrm{~m} 13.033 \mathrm{~s}$, DEC.$=+1 \mathrm{~d} 40 \mathrm{~m} 36.14 \mathrm{~s})$. While the 11 and $18 \mu \mathrm{m}$ images only show the northern side of the outflow, the FORCAST 31 and $37 \mu \mathrm{m}$ images also reveal the fainter southern outflow cavity. This cavity is also seen in the IRAC images, again being fainter than the northern side.

This trend agrees with the results of the RT simulations by ZT11 and ZTM13, which showed that at $\sim 40 \mu \mathrm{m}$, the near-facing outflow cavity is the dominant feature but, because of lower extinction, the structure along the outflow axis is more symmetric than at shorter wavelengths. In these models, the far-facing outflow appears in the NIR mainly due to the scattering by dust in and around the outflow cavity.

With their high dynamic range, the FORCAST images also show extended emission on larger scales, which is missing in the ground-base MIR images. The morphology of this extended emission has a resemblance to that seen with IRAC. This extended emission seems to be related to the cavity wall and/or a wider-angle part of the outflow. It is also possible that some, probably small, fraction of this emission is produced by other luminosity sources.

\subsection{SED and Protostellar Model Global Parameters}

We integrated over a $40^{\prime \prime} \times 50^{\prime \prime}$ region to find total 31 and $37 \mu \mathrm{m}$ fluxes of 527 and $922 \mathrm{Jy}$, respectively. No significant emission was seen outside of this area in either band. We constructed the SED using data from 2MASS (Skrutskie et al. 2006), GLIMPSE, Gemini-TReCS, IRAS-IGA (Cao et al.|1997), Herschel-SPIRE and JCMT-SCUBA (this paper), and IRAM 30m Telescope (Mooney et al. 1995). Total 2MASS and IRAC fluxes were integrated from background-subtracted images in the same region as the FORCAST bands. The 10 and $18 \mu \mathrm{m}$ fluxes (3.06 and 46.87 Jy, De Buizer 2006) were integrated with a $30^{\prime \prime}$ aperture (again, there are no significant sources in the immediate vicinity). For IRAS bands, the beams are so large that the $40^{\prime \prime} \times 50^{\prime \prime}$ region does not contain all the flux from this source, so

\footnotetext{
${ }^{1}$ Guest user, Canadian Astronomy Data Center
} 


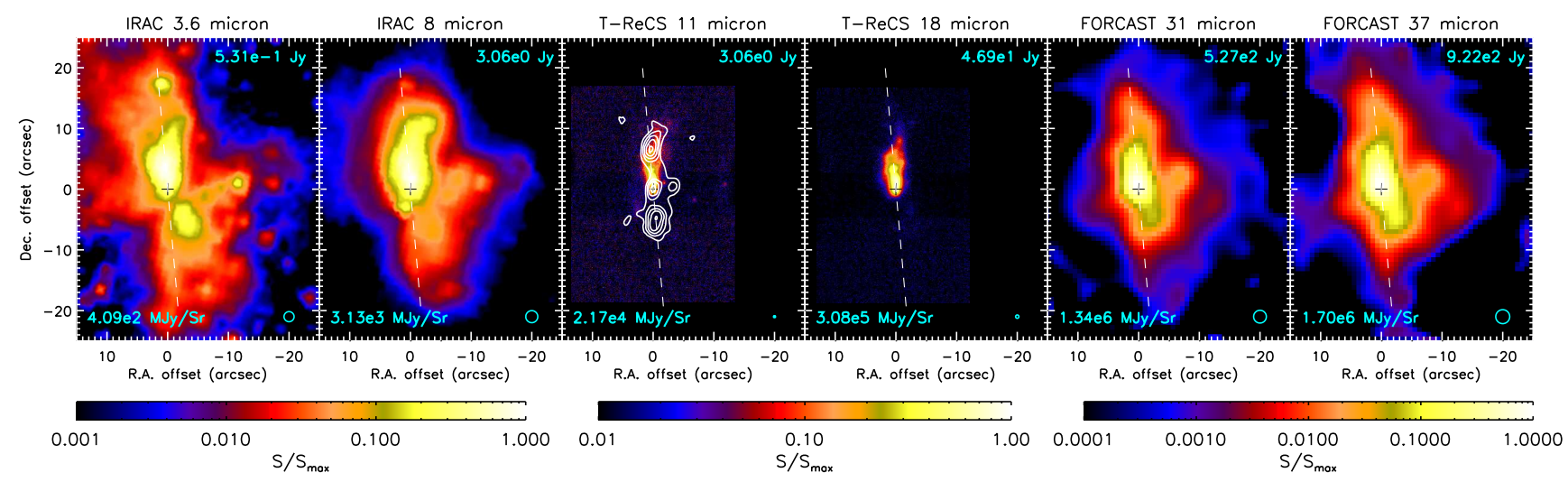

FIG. 1. - Multiwavelength images of G35.2. From left to right, IRAC (GLIMPSE) at 3.6 and $8 \mu \mathrm{m}$; T-Recs at 11 and $18 \mu \mathrm{m}$ (De Buizer 2006); (deconvolved) FORCAST at 31 and $37 \mu \mathrm{m}$. The white contours in the $11 \mu \mathrm{m}$ image show $15 \mathrm{GHz}$ radio continuum emission (Heaton \& Little 1988). Total fluxes are labeled in the upper-right corners and maximum surface brightnesses in the lower-left. The dashed line indicates the axis of the outflow deduced from the MIR and radio emission. Circles indicate image resolutions.

TABLE 1

PARAMETERS OF THE FOUR FITTED MODELS.

\begin{tabular}{c|c|c|c|c|c|c|c}
\hline Model & $\begin{array}{c}\text { Core mass } \\
M_{c}\left(M_{\odot}\right)\end{array}$ & $\begin{array}{c}\text { Mean surface density of } \\
\left.\text { the clump } \Sigma_{\mathrm{cl}}(\mathrm{g} \mathrm{cm})^{-2}\right)\end{array}$ & $\begin{array}{c}\text { Protostellar } \\
\text { mass, } m_{*}\left(M_{\odot}\right)\end{array}$ & $\begin{array}{c}\text { Outflow opening } \\
\text { angle } \theta_{w, \text { esc }}\end{array}$ & $\begin{array}{c}\text { Inclination } \\
\theta_{\text {view }}{ }^{\text {a }}\end{array}$ & $\begin{array}{c}\text { Foreground } \\
\text { extinction } A_{V}\end{array}$ & $\begin{array}{c}\text { Luminosity } \\
L_{\mathrm{bol}}\left(L_{\odot}\right) \mathrm{b}\end{array}$ \\
\hline Model 1 & 240 & 1 & 34 & $51^{\circ}$ & $58^{\circ}$ & 8 & $2.2 \times 10^{5}$ \\
\hline Model 2 & 240 & 0.7 & 26 & $45^{\circ}$ & $51^{\circ}$ & 8 & $1.2 \times 10^{5}$ \\
\hline Model 3 & 240 & 0.7 & 22 & $40^{\circ}$ & $44^{\circ}$ & 15 & $9.0 \times 10^{4}$ \\
\hline Model 4 & 240 & 0.4 & 20 & $35^{\circ}$ & $43^{\circ}$ & 0 & $6.6 \times 10^{4}$ \\
\hline
\end{tabular}

anclination between the line of sight and the outflow axis.

b True bolometric luminosity after allowing for foreground extinction and flashlight effect.

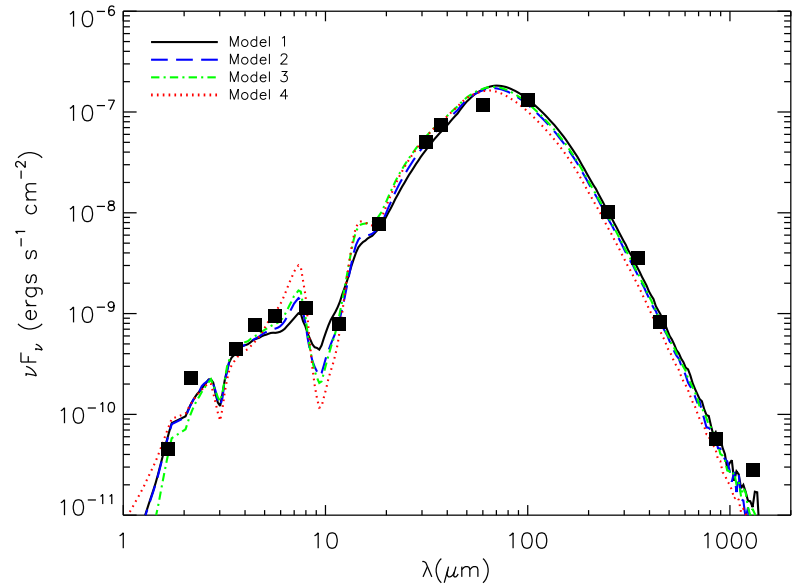

FIG. 2.- SED of G35.2 (solid squares) with observed fluxes from 2MASS H \& K bands, IRAC from 3.5 to $8 \mu \mathrm{m}$, T-ReCs $11,18 \mu \mathrm{m}$, FORCAST 31, $37 \mu \mathrm{m}$, IRAS 60, $100 \mu \mathrm{m}$, SPIRE 250, $350 \mu \mathrm{m}$, SCUBA 450, $850 \mu \mathrm{m}$, and IRAM $1.3 \mathrm{~mm}$. The lines show four model SEDs (see text).

we use larger apertures $\left(6.5^{\prime} \times 3^{\prime}\right)$ that encircle all the emission from the source while avoiding fluxes from significant nearby sources. We obtained fluxes at 60 and $100 \mu \mathrm{m}$ of $2.37 \times 10^{3}$ and $4.35 \times 10^{3} \mathrm{Jy}$. They may be considered as upper limits. For SPIRE and SCUBA images, we have carefully subtracted the backgrounds with two or more component Gaussian fitting. The fluxes are $546,420,123$, and $16 \mathrm{Jy}$ at 250, 350, 450, and $850 \mu \mathrm{m}$ respectively with source sizes of $27^{\prime \prime} \times 18^{\prime \prime}, 25^{\prime \prime} \times 3.8^{\prime \prime}$ at 250 and $350 \mu \mathrm{m}$, and $22^{\prime \prime} \times 12^{\prime \prime}$ in SCUBA bands. The SED is shown in Fig. (2).

We use the SED to constrain the properties of the protostar, its core and the surrounding clump by fitting with the RT models of ZT11 and ZTM13. The models are developed self-consistently for a core embedded in a high pressure clump forming a massive star via core accretion (MT03), including a treatment of rotating infall, an active accretion disk and a disk wind filling the outflow cavity. We have further extended the ZTM13 models to also include emission from the surrounding clump (see Fig. (3). Since we have not yet constructed a full model grid, we limit ourselves to achieving only a moderately good fit by exploring the parameters manually and with a quite coarse sampling of parameter space. We show four models that have relatively good matches to the SED in Fig. (2) with their parameters listed in Tab. (1).

Model 1 (Fig. 3) contains a core of initial mass $M_{c}=$ $240 M_{\odot}$, and a protostar of mass $m_{*}=34 M_{\odot}$ forming at its center. The core is embedded in a clump with $\Sigma_{\mathrm{cl}}=1 \mathrm{~g} \mathrm{~cm}^{-2}$, thus setting its radius $R_{c}=0.11 \mathrm{pc}$ (MT03). We distribute the clump material between 1 and $10 R_{c}$ with a power-law density profile (with index of 

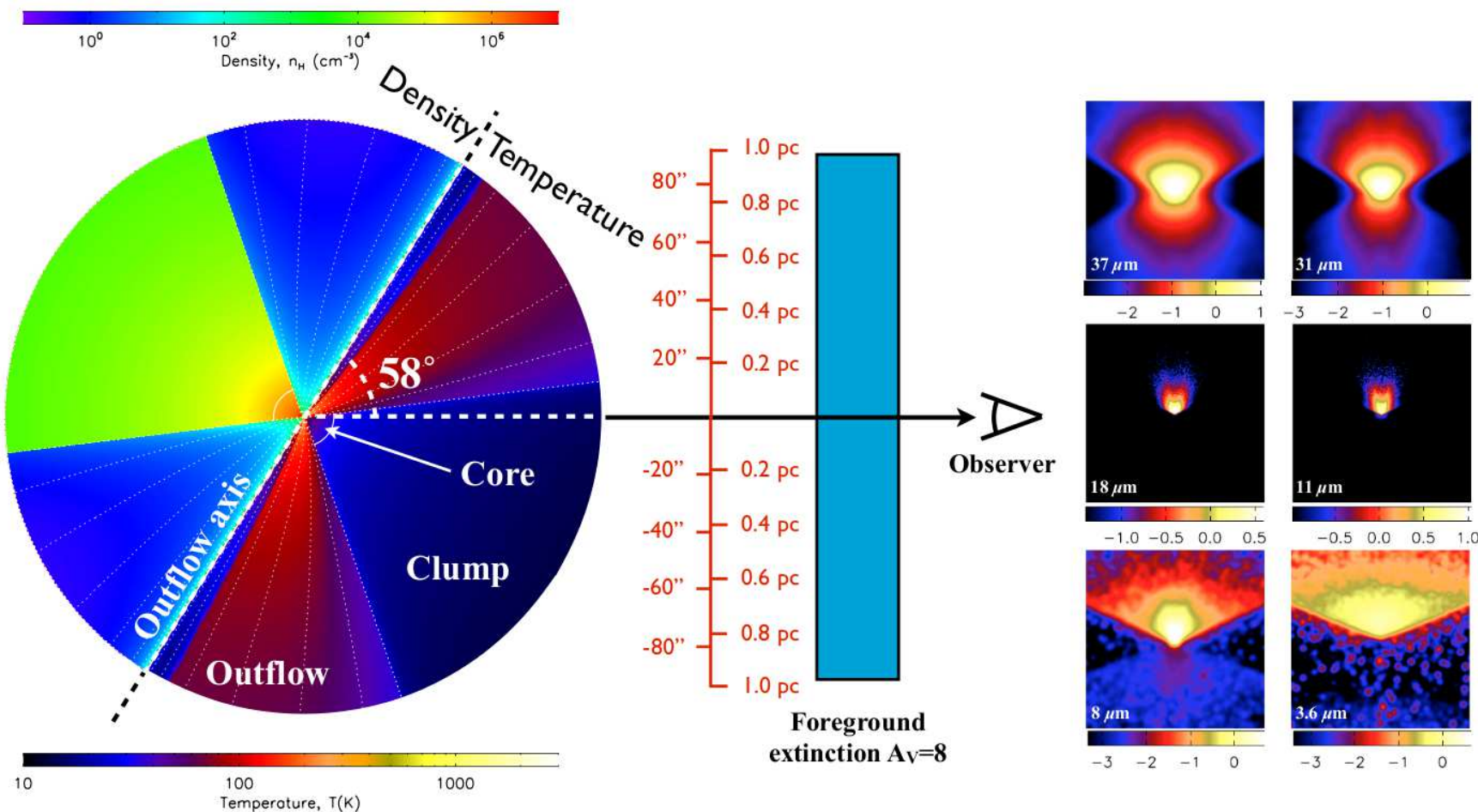

FIG. 3.- Cartoon showing geometry of Model 1. Density and temperature profiles are on the upper-left and lower-right sides of the outflow axis, respectively. White dotted lines show disk wind streamlines. The low temperature region close to the outflow axis is dust-free wind launched from the inner hot dust-free disk. The clump and the outflow extend to 10 core radii. The simulated images $\left(60^{\prime \prime} \times 60^{\prime \prime}\right)$ of Model 1 are also shown. The scalebars are in $\log \left(S / S_{\text {avg }}\right)$, where $S_{\text {avg }}$ is the average intensities of the near-facing outflow in each band (see Section 3.2). For the T-ReCs and FORCAST bands, we apply same dynamic ranges as in Fig. (1), while for the IRAC bands, we adopt larger dynamic ranges to show the far-facing outflow.

-1.75, e.g., Mueller et al. 2002) that joins the core boundary smoothly and reaches $\Sigma_{\mathrm{cl}} \simeq 1 \mathrm{~g} \mathrm{~cm}^{-2}$ by $10 R_{c}$ (counting two sides along a central sight line). Then the original clump mass (assuming spherical symmetry, i.e. before some material is swept-up by the outflow) is $4.8 \times 10^{3} M_{\odot}$. The size of the disk is set by assuming a ratio of rotational to gravitational energy of $2 \%$, yielding an outer radius of $940 \mathrm{AU}$. Its mass is assumed to be $1 / 3$ of the stellar mass. The accretion rate is $5.1 \times 10^{-4} M_{\odot} \mathrm{yr}^{-1}$. The total luminosity is $2.2 \times 10^{5} \mathrm{~L} \odot$ including protostar, accretion boundary layer and disk. An outflow cavity, filled with a disk wind, including dust along streamlines launched from the cooler disk outside of $7 \mathrm{AU}$, is included. Following ZT11 and ZTM13, the cavity opening angle, $\theta_{w \text {,esc }}=51^{\circ}$, to yield a final star formation efficiency from the core of $50 \%$. The viewing angle between the sight line and the outflow axis is $\theta_{\text {view }}=58^{\circ}$. At this viewing angle, if the received flux was assumed to be isotropic then a bolometric luminosity of $3.6 \times 10^{4} L_{\odot}$ would be implied. After considering the foreground extinction as a free parameter to better fit the NIR data $\left(A_{V}=8 \mathrm{mag}\right.$ for Model 1) and applying an appropriate aperture size $\left(\sim 50^{\prime \prime}\right)$, the inferred isotropic bolometric luminosity based on the received flux becomes $3.3 \times 10^{4} L_{\odot}$. Three other models with smaller values of $\Sigma_{\mathrm{cl}}, m_{*}$ and $\theta_{\text {view }}$ are also shown in Fig. (2) (see also Table (1).

Overall, good agreement is achieved between these model SEDs and the observations. Small discrepancies in the NIR and IRAC bands may be due to patchy ex- tinction and/or poorly-modeled polycyclic aromatic hydrocarbon (PAH) features and/or thermal emission from transiently heated small dust grains. At $60 \mu \mathrm{m}$ the observed IRAS flux is lower than the model (after convolving with the relatively broad filter response of IRAS $60 \mu \mathrm{m}$ band) by $\sim 25 \%$. These results show the SED fitting has a certain degeneracy in model parameter space, especially when the opening angle is not well constrained (ZTM13 found the outflow, especially the bright part, at 10 to $20 \mu \mathrm{m}$ appears narrower than the actual cavity, so the MIR morphology may not be a reliable tracer of the total outflow opening angle). Models with narrower outflow cavities reprocess larger fractions of their luminosities and thus have smaller bolometric flashlight effect corrections for the considered viewing angles. Thus lower luminosity protostars are able to reproduce the observed FIR peak of the SED. Then, lower values of $\Sigma_{\mathrm{cl}}$ and $\theta_{\text {view }}$ are needed to match the MIR fluxes. Observations at $10 \mu \mathrm{m}$ with higher spectral resolution will provide strong diagnostics for testing these models. However, even with a large uncertainty in the outflow opening angle, the protostellar mass is still estimated to be $\sim 20-34 M_{\odot}$ and $\Sigma_{\mathrm{cl}} \sim 0.4-1 \mathrm{~g} \mathrm{~cm}^{-2}$, i.e., G35.2 is a massive protostar forming from a massive core embedded in a high surface density clump.

\subsection{Resolved Intensity Profiles Along the Outflow Axis}

The images of Model 1 are shown in Fig. (3) for reference. The other three models selected from SED fitting show slightly narrower outflow cavities. We compare the 


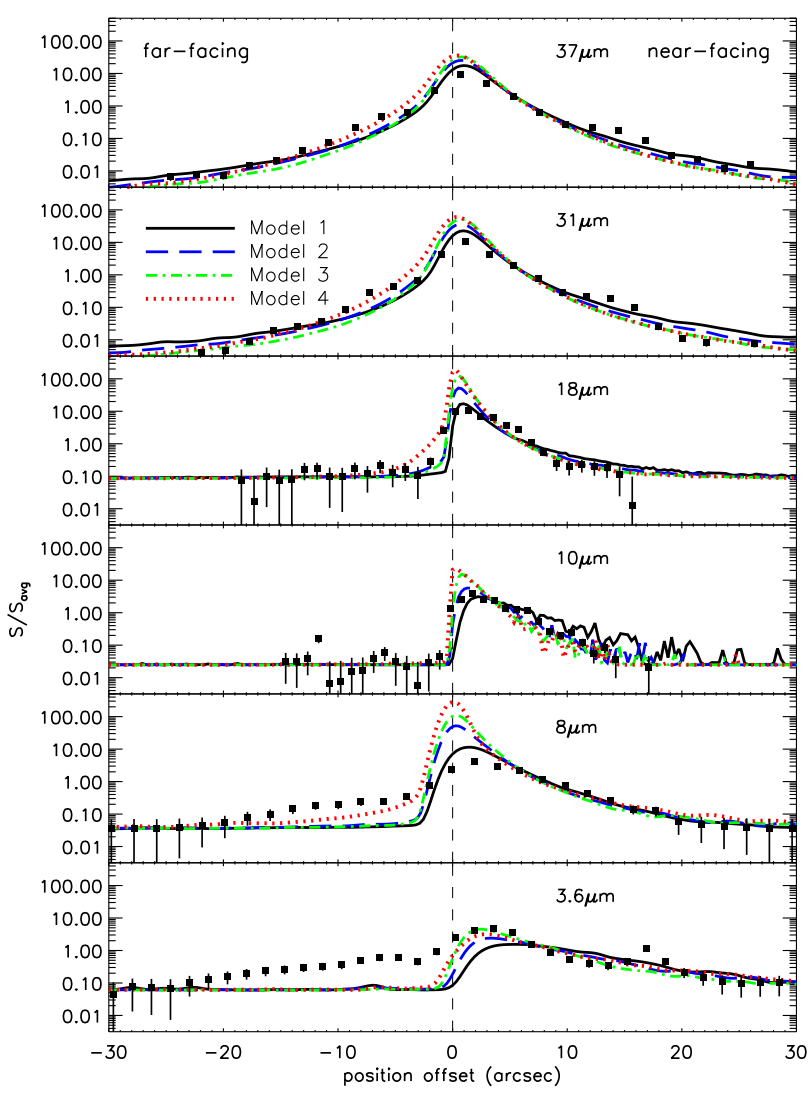

Fig. 4.- Intensity profiles along the outflow axis. The squares are observational data sampled at intervals of the resolutions of the instruments (intervals of $2 \& 3 \times$ resolution are used for 18 \& $10 \mu \mathrm{m}$ ) with errors composed of systematic flux uncertainties (assumed to be 20\%) and estimated background noise. The lines are model profiles.

intensity distribution along the outflow axis predicted by these models with observations in Fig. (4)). The axis direction of G35.2 is chosen via the radio continuum and MIR morphology (dashed line in Fig. (1) with P.A. of $6^{\circ}$ ). The model profiles are all convolved with the corresponding instrument beams. At each offset from the center, we average over a perpendicular width of $2^{\prime \prime}$ to estimate profiles for both model and data. All model and observed profiles are normalized to the average values over the near-facing regions that have significant emission. We also add constant background ambient intensities, which may be either due to instrumental noise (i.e. in the T-ReCS data) or from additional ambient interstellar material. Note we have not attempted to use the lateral width of the cavities to constrain the models

We emphasize that a detailed model search to fit these profiles separately from the SED has not been performed, but still Model 1 agrees very well with the observations at $37,31,18$ and $10 \mu \mathrm{m}$, producing the right peak positions and asymmetries of the two sides of the outflow. At $10 \mu \mathrm{m}$, Model 1 predicts higher extinction towards the center. A possible explanation could be the strength (depth and width) of the silicate absorption feature adopted in the dust model used in the RT calculations (ZTM13). In the IRAC bands, while a reasonable fit is achieved for the near-facing outflow cavity, the in- tensities of the far-facing side predicted by the model are too low compared to those observed. Possible reasons for this include: (1) non-uniform, possibly patchy, extinction from the clump or the adopted uniform "foreground" extinction, that may actually be relatively local to the source (e.g. a foreground extinction of $A_{V}=15$ lowers $3.6 \mu \mathrm{m}$ flux by a factor of two); (2) enhanced emission from PAHs and transiently-heated small dust grains in and around the outflow cavity. Models 2, 3 and 4 also have good matches with the data in the near-facing and far-facing wings at 37, 31, 18 and $10 \mu \mathrm{m}$, but their profiles become too peaked towards the center due to the lower extinction of their lower $\Sigma$ cores and clumps.

These results, especially the intensity profiles for Model 1 from $\sim 10-40 \mu \mathrm{m}$ (for which the modeling has potentially fewer problems associated with small grain and PAH emission), again support the paradigm that G35.2 is a massive protostar forming from a high $\Sigma$ core and clump. The match of the SED and intensity profiles with observations becomes less good when $\Sigma_{\mathrm{cl}}$ becomes lower. We estimate that $\Sigma_{\mathrm{cl}}$ should be no lower than $0.4 \mathrm{~g} \mathrm{~cm}^{-2}$. The profiles also suggest that the accretion flow and outflows are relatively well-ordered and symmetric.

\section{DISCUSSIONS \& CONCLUSIONS}

SOFIA-FORCAST provides imaging with high dynamic range similar to space-based instruments at a unique wavelength region $\sim 30$ to $40 \mu \mathrm{m}$, where lower extinction allows us to search for the predicted (ZT11, ZTM13) far-facing outflow cavity from a massive protostar forming from a high surface density core. At longer wavelengths the emission is predicted to become even more symmetric, being dominated by cooler dust in the core/clump. Our SOFIA-FORCAST observations at 31 and $37 \mu \mathrm{m}$ did reveal emission from the far-facing outflow cavity of G35.2, which was too faint to detect by ground-based T-ReCs 11 and $18 \mu \mathrm{m}$ observations.

We compiled the NIR to mm SED of G35.2. RT modeling of a massive protostar forming from a massive core bounded by a high $\Sigma$ clump gave good agreement with this SED for four models. Depending on the outflow cavity opening angle $\left(35^{\circ}\right.$ to $\left.50^{\circ}\right)$, we found $m_{*} \sim 22-34 M_{\odot}$, $L_{\text {bol }} \sim(0.7-2.2) \times 10^{5} L_{\odot}$ and $\Sigma_{\mathrm{cl}} \sim 0.4-1 \mathrm{~g} \mathrm{~cm}^{-2}$. Model 1 also produced intensity profiles along the outflow axis that fit the observations well at 10, 18, 31 and $37 \mu \mathrm{m}$, without need for extensive fine tuning. These results indicate G35.2 is a massive protostar, forming from high surface density core and clump, via relatively ordered, symmetric collapse and accretion. Powerful bipolar outflows are being launched and have cleared wideangle cavities, that are also relatively symmetric.

A protostar with the luminosity estimated here $(\sim$ $\left.1 \times 10^{5} L_{\odot}\right)$ is expected to drive a $\mathrm{CO}$ outflow with momentum flux of $\sim 0.1 M_{\odot} \mathrm{yr}^{-1} \mathrm{~km} \mathrm{~s}^{-1}$ (Richer et al. 2000), which is much larger than the observed value: even assuming that the larger NE-SW CO outflow is also driven by this source, the total momentum flux is still only $\sim 0.003 M_{\odot} \mathrm{yr}^{-1} \mathrm{~km} \mathrm{~s}^{-1}$ (Gibb et al. 2003, Birks et al. 2006). This may indicate that the outflow from G35.2N, being partly ionized as indicated by the observed radio continuum jet, may be relatively deficient in $\mathrm{CO}$ emission. Note that the modest misalignment of the larger $\mathrm{CO}$ outflow with the radio and MIR jet may result from the interaction of the wider angle part of the 
outflow with a core/clump that does have some moderate lateral asymmetries.

We conclude that, at least in the case of G35.2, massive star formation appears to be well described by predictions of the Turbulent Core Model (MT03), even with a highly idealized realization of the model utilizing perfect axissymmetry and smooth density structures (see Cunningham et al. 2011 for a numerical simulation of this model including outflows). Such ordered accretion and outflow is not expected in models involving massive star formation via competitive accretion or stellar collisions, nor indeed in cores that are highly turbulent. This may indicate that magnetic fields are dynamically important in setting the structure of the core.
This work is based on observations made with the NASA/DLR Stratospheric Observatory for Infrared Astronomy (SOFIA). SOFIA is jointly operated by the Universities Space Research Association, Inc. (USRA), under NASA contract NAS2-97001, and the Deutsches SOFIA Institut (DSI) under DLR contract 50 OK 0901 to the University of Stuttgart. YZ acknowledges support from a Graduate School Fellowship from the Univ. of Florida. JCT acknowledges support from NSF CAREER grant AST-0645412 and NASA/USRA grant in support of SOFIA Basic Science observations. CFM acknowledges support from NSF grants AST-0908553 and AST-1211729 and NASA grant NNX09AK31G.

\section{REFERENCES}

Adams, F. C., 2010, ARAA, 48, 47

Beuther, H., Schilke, P., Gueth, F., McCaughrean, M., Andersen, M., Sridharan, T. K., Menten, K. M., 2002, A\&A, 387, 931

Birks, J. R., Fuller, G. A., Gibb, A. G., 2006, A\&A, 458, 181

Bonnell, I. A., Bate, M. R., Clarke, C. J., Pringle, J. E., 2001, MNRAS, 323, 785

Bonnell, I. A., Bate, M. R., Zinnecker, H., 1998, MNRAS, 298, 93

Butler, M. J., Tan, J. C., 2012, ApJ, 754, 5

Cao, Y., Terebey, S., Prince, T. A., Beichman, C. A., 1997, ApJS, 111,387

Churchwell, E., Babler, B. L., Meade, M. R., et al., 2009, PASP, 121,213

Cunningham, A. J., Klein, R. I., Krumholz, M. R., McKee, C. F., 2011, ApJ, 740, 107

De Buizer, J. M., 2006, ApJL, 642, 57

De Buizer, J. M., Bartkiewicz, A., Szymczak, M., 2012, ApJ, 754, 149

Dent, W. R. F., Little, L. T., Kaifu, N., Ohishi, M., Suzuki, S., 1985, A\&A, 146, 375

Fuller, G. A., Zijlstra, A. A., Williams, S. J., 2001, ApJL, 555, 125

Gibb, A. G., Hoare, M. G., Little, L. T., Wright, M. C. H., 2003 , MNRAS, 339, 1011

Heaton, B. D., Little, L. T., 1988, A\&A, 195, 193

Herter, T. L., Adams, J. D., De Buizer, J. M., Gull, G. E., Schoenwald, J., Henderson, C. P., Keller, L. D., Nikola, T., Stacey, G., Vacca, W. D., 2012, ApJL, 749, 18

Little, L. T., Kelly, M. L., Murphy, B. T., 1998, MNRAS, 294, 105

Lucy, L. B., 1974, AJ, 79, 745
Matzner, C. D., McKee, C. F., 1999, ApJ, 526, L109

McKee, C. F., Tan, J. C., 2003, ApJ, 585, 850

Mooney, T., Sievers, A., Mezger, P. G., Solomon, P. M., Kreysa, E., Haslam, C. G. T., Lemke, R., 1995, A\&A, 299, 869

Mueller, K. E., Shirley, Y. L., Evans, N. J., II, Jacobson, H. R., 2002, ApJS, 143, 469

Paron, S., Weidmann, 2010, MNRAS, 2010, 408, 2487

Richardson, W. H., 1972, Opt. Soc. Am., 62, 55

Richer, J. S., Shepherd, D. S., Cabrit, S., Bachiller, R., Churchwell, E., 2000, in Protostars and Planets IV, ed. V. Mannings, A. P. Boss, \& S. S. Russell (Tucson, AZ: Univ. Arizona Press), 867

Sandell, G., Jessop, N., Jenness, T. 2001, The SCUBA Map Reduction Cookbook, Starlink Cookbook 11.2, Rutherford Appleton Laboratory, Particle Physics \& Astronomy Research Council

Skrutskie, M. F. et al., 2006, ApJ, 131, 1163

Tan, J. C., McKee, C. F., 2003, arXiv:astro-ph/0309139v1]

Wang, P., Li, Z.-Y., Abel, T., Nakamura, F', 2010, ApJ, 709, 27

Whitney, B. A., Wood, K., Bjorkman, J. E., Wolff, M. J., 2003, ApJ, 591, 1049

Zhang, Y., Tan, J. C., 2011, ApJ, 733, 55

Zhang, Y., Tan, J. C., McKee, C. F., 2013, accepted to ApJ, arXiv:astro-ph/1212.3899

Zhang, B., Zheng, X. W., Reid, M. J., Menten, K. M., Xu, Y., Moscadelli, L., Brunthaler, A., 2009, ApJ, 693, 419 